Genomics

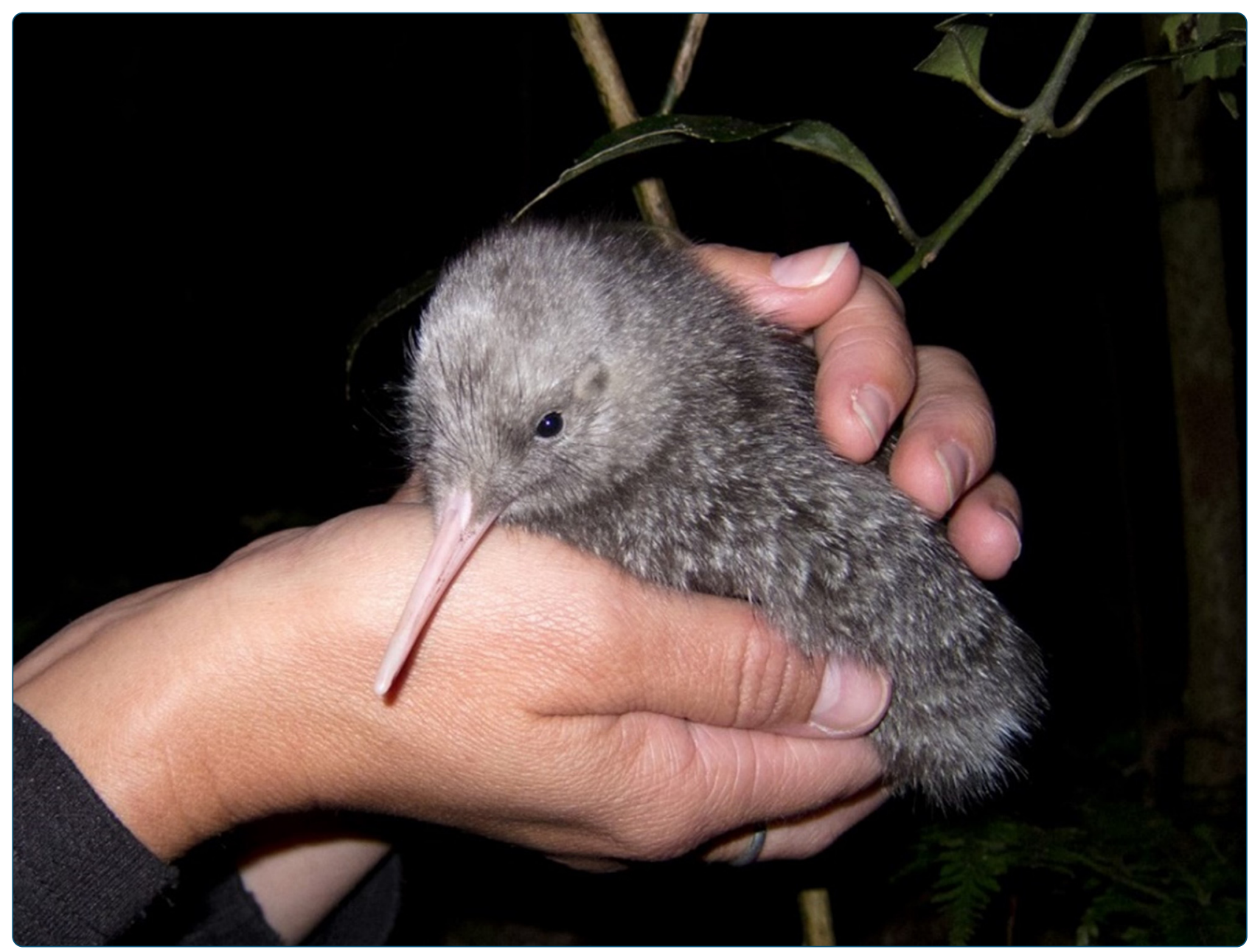

Sixteen kiwi (Apteryx spp) transcriptomes provide a wealth of genetic markers and insight into sex chromosome evolution in birds

Ramstad et al.

C Biomed Central 


\title{
Sixteen kiwi (Apteryx spp) transcriptomes provide a wealth of genetic markers and insight into sex chromosome evolution in birds
}

Kristina M. Ramstad ${ }^{1,2^{*}}$, Hilary C. Miller ${ }^{3}$ and Gabriel Kolle ${ }^{4}$

\begin{abstract}
Background: Kiwi represent the most basal extant avian lineage (paleognaths) and exhibit biological attributes that are unusual or extreme among living birds, such as large egg size, strong olfaction, nocturnality, flightlessness and long lifespan. Despite intense interest in their evolution and their threatened status, genomic resources for kiwi were virtually non-existent until the recent publication of a single genome. Here we present the most comprehensive kiwi transcriptomes to date, obtained via Illumina sequencing of whole blood and de novo assembly of mRNA sequences of eight individuals from each of the two rarest kiwi species, little spotted kiwi (LSK; Apteryx owenii) and rowi (A. rowi).
\end{abstract}

Results: Sequences obtained were orthologous with a wide diversity of functional genes despite the sequencing of a single tissue type. Individual and composite assemblies contain more than 7900 unique protein coding transcripts in each of LSK and rowi that show strong homology with chicken (Gallus gallus), including those associated with growth, development, disease resistance, reproduction and behavior. The assemblies also contain 66,909 SNPs that distinguish between LSK and rowi, 12,384 SNPs among LSK (associated with 3088 genes), and 29,313 SNPs among rowi (associated with 4953 genes). We found 3084 transcripts differentially expressed between LSK and rowi and 150 transcripts differentially expressed between the sexes. Of the latter, 83 could be mapped to chicken chromosomes with 95\% syntenic with chromosome Z.

Conclusions: Our study has simultaneously sequenced multiple species, sexes, and individual kiwi at thousands of genes, and thus represents a significant leap forward in genomic resources available for kiwi. The expression pattern we observed among chromosome $Z$ related genes in kiwi is similar to that observed in ostriches and emu, suggesting a common and ancestral pattern of sex chromosome homomorphy, recombination, and gene dosage among living paleognaths. The transcriptome assemblies described here will provide a rich resource for polymorphic marker development and studies of adaptation of these highly unusual and endangered birds.

Keywords: Transcriptome, Kiwi, Apteryx, Sex chromosome evolution, De novo assembly

\footnotetext{
* Correspondence: kristinar@usca.edu

'Department of Biology and Geology, University of South Carolina Aiken, 471

University Parkway, Aiken, SC 29801, USA

${ }^{2}$ Allan Wilson Centre, School of Biological Sciences, PO Box 600, Victoria

University of Wellington, Wellington 6140, New Zealand

Full list of author information is available at the end of the article
} 


\section{Background}

Species of conservation concern have arguably the greatest need for genomic tools but can present significant challenges to their development and use. Sampling large numbers of individuals or obtaining fresh tissue samples is often impossible when working with at-risk species due to their rarity and protected status $[1,2]$. The species of interest may also be phylogenetically divergent and lack a closely related reference genome, making genomic annotation and assembly difficult [3]. In addition, many at-risk populations will have experienced genetic bottleneck effects, small population size, and inbreeding, which can result in low levels of genetic diversity and strong gametic disequilibrium and make discovery of large numbers of independent polymorphic markers challenging. These difficulties initially slowed the development and use of genomic tools for endangered species, and the full application and power of conservation genomics to improve species management is only now becoming evident [4-9]. At the same time, the field of genomics has placed emphasis on broad evolutionary comparisons rather than on delineating genomic variation within species. Genomic studies often deeply sequence one to three individuals of a single species for use in comparisons among orders or families. Conservation management, however, occurs at the population level, with a focus on understanding and maintaining genetic diversity within and between species.

Transcriptome sequencing allows elucidation of coding gene sequences without the requirement of deep genome sequencing. Next-generation sequencing techniques allow for the collection of mRNA sequences from small amounts of tissue in parallel across individuals and species [2], at a depth and coverage that allow de novo transcriptome assembly even in the absence of a closely related genome [10]. Tissue samples can be collected non-lethally from a single tissue type, such as skin, and still contain sequences from large numbers of genes with diverse functions [2]. Alternatively, samples from diverse tissues can be taken from individuals that have recently died of natural causes, and still provide enough high quality sequence data to build a transcriptome [1]. This provides exciting new possibilities for transcriptome assembly and characterization from rare and endangered species, and thus unprecedented possibilities for marker discovery, gene characterization, and comparative genomics of these species as well.

Kiwi (Family Apterygidae) are ratite birds endemic to New Zealand. They are of great evolutionary interest because they represent the most basal extant avian lineage (paleongaths) and possess numerous biological attributes that are unusual or extreme among birds. These include large egg size, paired functional ovaries, low metabolic rate, strong olfaction, and lack of color vision $[11,12]$ as well as flightlessness (present in $<1 \%$ of extant birds) [13], long lifespan (potentially more than 80 years) [14$16]$, and nocturnality (less than $3 \%$ of avian species) [11, 17]. Kiwi are also of great conservation concern, with four of their five species listed as threatened by the International Union for Conservation of Nature [18]. Kiwi comprise two clades, the brown and spotted kiwi, which diverged from one another approximately 16 million years ago [19]. The rowi (Apteryx rowi) is a brown kiwi and the rarest kiwi species. They comprise a single population of approximately 430 birds that rebounded from a population size of approximately 150 birds in the mid 1990's [20]. A third of adult rowi do not breed and annual hatching success in the wild is $16 \%$ at best [21]. The little spotted kiwi (LSK, A. owenii) is the second rarest kiwi species. All extant LSK arose from at most five birds placed on Kapiti Island approximately 100 years ago. Despite rapid population growth, LSK are depauperate in genetic diversity at important disease resistance genes [22], show signs of inbreeding depression [23], and continue to experience genetic erosion [24]. Annual hatching success for wild LSK can be as low as 27\% [23]. Reduced fertility and hatching are often the first signs of inbreeding depression in small, isolated avian populations [25-29]. Thus, poor reproductive success may have an underlying genetic basis in LSK and rowi, potentially exacerbated by the strong genetic drift they experienced during recent genetic bottlenecks [24] and subsequent inbreeding due to small population size [23].

Intense conservation measures have been taken with kiwi, including captive incubation, translocation, and predator control programs [20]. Genomics research could greatly enhance these efforts by providing robust pedigrees, assessing levels of hybridization, investigating possible genotype-phenotype correlations and estimating relatedness and inbreeding coefficients. However, few genomic resources have been available for kiwi until recently $[11,30]$ and none have sequenced more than three birds representing a single species and sex. Indeed, all genomic studies of kiwi to date have focused on the relatively common ( 25,000 individuals) and widespread North Island brown kiwi (NIB, A. mantelli) [20] without regard to the genetic divergence expected between clades or species.

In this study, we used Illumina sequencing to assemble transcriptomes of 16 kiwi solely from samples of whole blood. Our primary objective was to define suites of functional and neutral genetic markers for brown and spotted kiwi in a single sequencing effort and without having to sacrifice any birds. Specifically, we sought to identify (1) markers in genes of known function that display coding DNA sequence variation between species and among individuals, (2) putatively neutral genetic markers that display DNA sequence variation between 
species and among individuals and (3) markers in genes that show differential expression between species and sexes. For each species, we assembled 200 million paired end reads into approximately 200 thousand contigs, of which approximately 20 thousand contained predicted coding gene sequences with mean open reading frame (ORF) lengths greater than 900bp. We were able to annotate more than half of these ORF containing contigs by comparison with sequences in the NCBI nonredundant protein database. In addition, we have catalogued an extensive list of sequence variation, found many genes that are differentially expressed between species and sexes, and identified polymorphic markers within genes related to reproduction, growth, development, behavior, and disease resistance. Our results will help delineate the structure, function and expression of kiwi genes, and provide a powerful resource for further studies of kiwi ecology, management, and evolution.

\section{Results and discussion}

\section{De novo assembly and annotation}

We sequenced mRNA libraries from the blood of eight LSK and eight rowi using an Illumina HiSeq and obtained more than 348 and 502 million paired reads from LSK and rowi, respectively. Raw HiSeq reads are available at the NCBI Short Read Archive under accession number SRP074490. While a more comprehensive transcriptome could have been obtained from sequencing mRNA from various tissues, our non-lethal sampling means that we can continue to monitor our study birds to explore additional genotype-phenotype relationships in the future. A possible limitation of sequencing only blood is the high level of hemoglobin transcripts often encountered $(50-70 \%)[31,32]$. However, we found that only $18 \%$ of read pairs $(n=150,157,518)$ in the final data $(n=$ $851,254,015)$ mapped to hemoglobin (Table 1), enabling us to still assemble comprehensive transcriptomes from a moderate number (>700 million in total) of reads.

Table 1 Summary of transcript assembly for LSK and rowi

\begin{tabular}{lll}
\hline & LSK & Rowi \\
\hline Total number of read pairs & $348,651,735$ & $502,602,280$ \\
Number of reads mapped to hemoglobin & $68,514,883$ & $81,642,635$ \\
Total number of contigs & 215,094 & 207,470 \\
Number of contigs with predicted ORF $>$ & 20,152 & 23,830 \\
100aa & & \\
Mean transcript length (ORF > 100aa) & $2,225 \mathrm{bp}$ & $2,847 \mathrm{bp}$ \\
Mean ORF length (ORF > 100aa) & $917 \mathrm{bp}$ & $1,129 \mathrm{bp}$ \\
Number of possible chicken orthologues & 7,908 & 8,631
\end{tabular}

Greater than 20 thousand contigs in each of LSK and rowi have a full or partial open reading frame (ORF) greater than 100 amino acids and thus a predicted protein coding sequence. Within species, approximately 8000 of these contigs show strong homology to chicken protein sequences
After removal of the transcripts mapping to hemoglobin, two hundred million reads from each species were randomly selected for Trinity assembly from those remaining, and formed 215,094 and 207,470 contigs for LSK and rowi, respectively (Table 1). Of these, 20,152 LSK and 23,830 rowi transcripts had a predicted full or partial Open Reading Frame (ORF) greater than 100 amino acids, which we focused our analyses on subsequently. Using BLASTP, we found 7908 transcripts in LSK and 8631 transcripts in rowi with strong homology $(>80 \%)$ to the chicken Ensemble protein database (Table 1).

BLASTN comparison determined that more than 16,000 contigs containing ORFs were shared between the two species with $99 \%$ average homology. Thus, the LSK composite assembly was used as the master reference in all subsequent analyses (Additional file 1). We found strong alignment between our composite LSK transcriptome and the recently published North Island brown kiwi genome [11]. Of our 20,152 transcripts, 97\% $(19,533)$ mapped to at least one contig from the genome assembly with $76 \%(15,315)$ mapping to greater than $90 \%$ of the entire contig length with $>99 \%$ identity.

Sequences were further annotated by searching the NCBI non-redundant (NR) protein database using BLASTx (Additional file 2). Of the 20,152 unique LSK contigs containing an annotated ORF, 12,571 (62\%) had matches with an e-value of less than $1 \mathrm{e}^{-4}$. Most of these matches were to birds and reptiles (Fig. 1), but a large diversity of species contributed to functional annotation. The highest number of top hits (alignments with the lowest e-value per sequence) were attributed to the mallard (Anas platyrhynchos, 1976 transcripts or 16\%), chicken (1593 or 13\%), peregrine falcon (Falco peregrinus, 1446 or 12\%) and pigeon (Columbia livia, 1422 or $11 \%)$. Secondly, we used Blast2GO to assign Gene Ontogeny (GO) identifiers to 10,688 of the 12,571 sequences for which we obtained BLASTx results, or 53\% of the 20,152 contigs originally blasted. More than half of the annotated genes were associated with biological regulation or cellular, metabolic, or single-organism processes (Fig. 2a). Greater than $80 \%$ of sequences annotated with a molecular function were associated with binding or catalytic activity (Fig. 2b), which is typical of transcriptomic studies regardless of taxa or tissue type sequenced $[1-3,30]$. Importantly, we identified numerous sequences associated with growth $(n=355)$, reproduction $(n=554)$ and development $(n=2239)$.

\section{Sequence variation between and within species}

One of the primary aims of this work was to define a highly confident sequence variant list that could be used for both population monitoring and genotype-phenotype associations in kiwi. To that end, we identified the full 


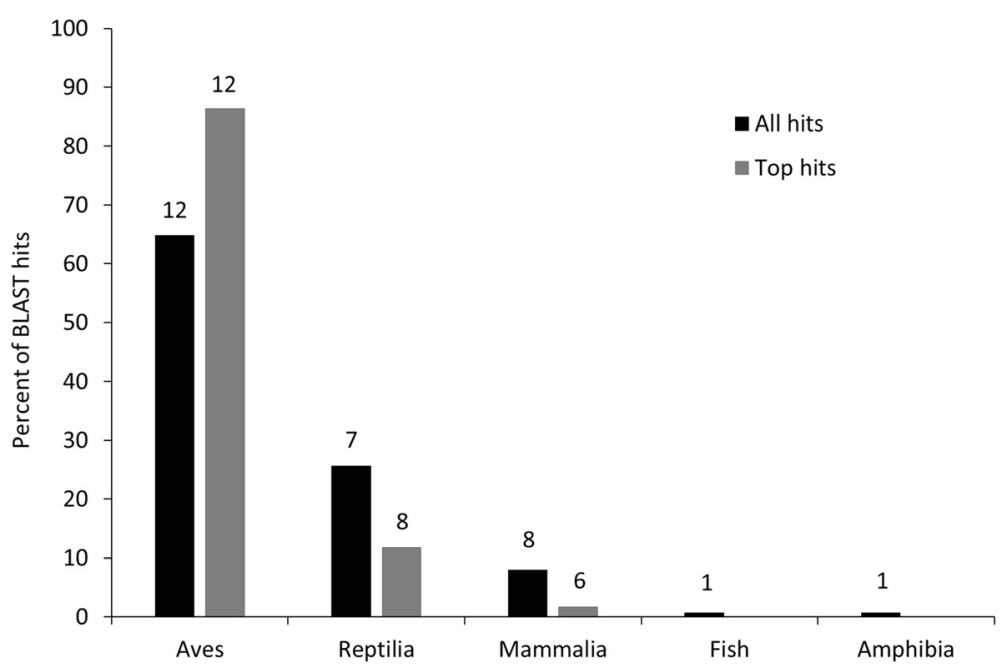

Fig. 1 Percent of BLAST hits of kiwi mRNA transcripts against NCBI non-redundant (NR) database by vertebrate class. Numbers above bars indicate number of species represented within the percentage. Amphibians were represented solely by African clawed frogs (Xenopus laevis) and fishes by the African coelacanth (Latimeria chalumnae)

cohort of SNP variation across all samples by comparison to the LSK reference assembly. We found 83,222 and 434,504 SNPs present in at least one LSK or rowi, respectively. To find markers with high confidence, we filtered for SNPs present in at least 50 reads per individual. This resulted in a total of 27,170 SNPs in LSK and 120,035 SNPs in rowi. Of the SNPs found in rowi, 66,909 in 6164 contigs were exclusive to rowi and differentiate between the two species (Table 2; Additional file 3). A total of 12,384 (in 3089 contigs) and 29,313 (in 4953 contigs) SNPs in LSK and rowi met our criteria for a marker that could distinguish individuals within each species: (a) placement within contigs where SNP density was less than 1 per 200bp and (b) at least one individual with a SNP frequency lower than $5 \%$ and one with a frequency greater than 35\% (Table 2; Additional files 4 and 5).

We further categorized SNPs based on their likely effect on the gene coding sequence, finding 5434 in LSK and 17,057 in rowi that result in changes to amino acid coding sequences (Table 2). These potential markers map to genes present in each of the chicken chromosomes (Additional files 3, 4 and 5) and are candidates for targets of selection. In contrast, many of the SNPs we discovered are likely selectively neutral because they are positioned in untranslated regions of contigs or cause synonymous substitutions in coding regions.

Polymorphism in rowi was more than double that observed among LSK. This pattern is not likely due to differences in sequencing depth as there is little correlation between the number of species specific SNPs and the total number of reads sequenced for any individual kiwi (Pearson correlation coefficient $=0.09$ ). More likely, the much greater SNP diversity detected in rowi, a pattern that has also been observed in mtDNA [33] and among microsatellite loci [34], reflects the differences in demographic history of the two species. LSK were reduced to five birds approximately 100 years ago and show numerous intense signs of this recent genetic bottleneck effect [24]. In contrast, rowi represent a very old and natural remnant population $[33,35]$ and though recently bottlenecked, retain greater ancestral genetic variation than LSK (K Ramstad, unpublished data) [34].

\section{Differential gene expression between species and sexes}

By mapping reads to the reference and counting the number of reads mapping to each transcript, we found significant differences in patterns of gene expression between kiwi species and sexes (Additional file 6). A total of 3084 transcripts were differentially expressed by at least 2 fold between LSK and rowi $(\beta$-statistic $>0)$, including 1753 upregulated in LSK and 1331 upregulated in rowi (Fig. 3a; Additional file 7). Mean upregulation of genes in LSK and rowi was 8.1 fold (range 2.0 to 701.0) and 5.0 fold (2.0 to 242.5), respectively. Biological process GO terms were assigned to 524 genes upregulated in LSK and 643 genes upregulated in rowi. The distribution of genes across GO terms (weighted by the number of genes annotated) was similar in the two species, with genes involved in cellular processes, localization, and signaling more prevalent among genes upregulated in LSK and genes involved in development and cellular component organization more prevalent among genes upregulated in rowi (Fig. 3b).

We did not use a 2 fold threshold in comparing expression differences between sexes as a 2 fold male-bias 


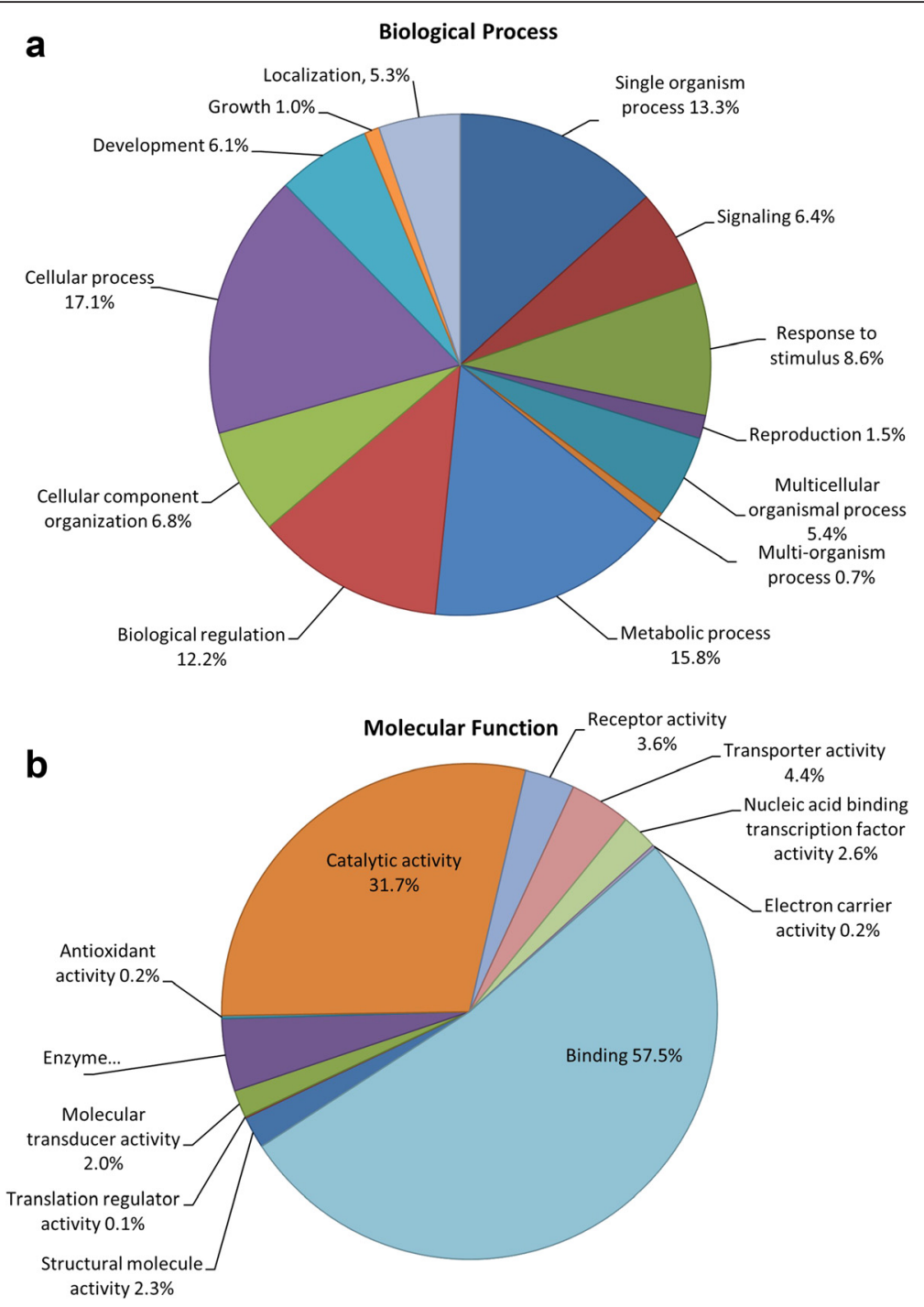

Fig. 2 Level 2 gene ontology assignments of (a) biological process and (b) molecular function for little spotted kiwi transcripts

is expected of genes present on the $\mathrm{Z}$ chromosome (chrZ) but outside the pseudoautosomal region (PAR). We identified 150 differentially expressed genes with a $\beta$-statistic $>0$ between sexes ( 88 of these with an expression difference $\geq 2$ fold). Of these genes, 94 showed male-biased (38 genes $>2$ fold difference) and 56 (50 genes >2fold difference) showed female biased expression (Fig. 4a; Additional file 8). Male-biased expression ranged from 1.4 to 3.8 fold with a mean of 2.0 (range 2.0 to 3.8 , mean 2.4 where expression $>2$ fold), while female-biased expression ranged from 1.6 to 101.4 fold with a mean

Table 2 Summary of SNPs discovered in LSK and rowi and those predicted to change protein coding sequences

\begin{tabular}{lll}
\hline & Total number & Number predicted to change amino acid \\
\hline All SNPs found in LSK & 27,170 & 5,434 \\
All SNPs found in rowi & 120,035 & 17,057 \\
SNPS that differentiate LSK and rowi & 66,909 & 7,418 \\
SNPs that differentiate among LSK & 12,384 & 1,832 \\
SNPS that differentiate among rowi & 29,313 & 4,466 \\
\hline
\end{tabular}

SNPs that are unique to rowi and differentiate between species have greater than $50 \%$ coverage in each bird sequenced, a frequency of greater than $80 \%$ among all rowi sequenced, and less than 5\% among sequenced LSK. SNPs that differentiate among LSK and rowi are within contigs with a SNP density less than 1 per $200 \mathrm{bp}$ and have a frequency of greater than $35 \%$ in at least one individual and less than $5 \%$ in another 


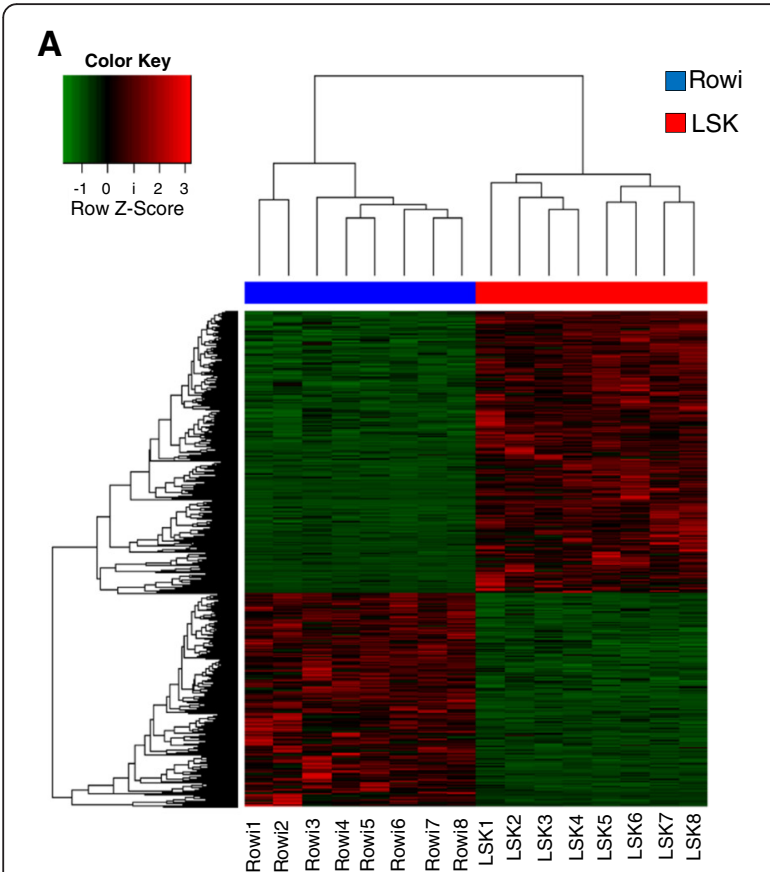

B

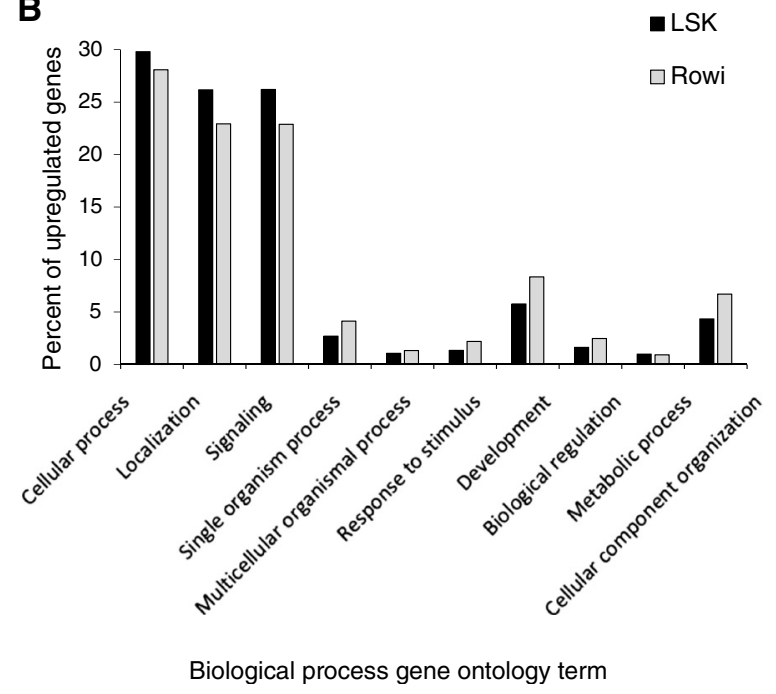

Fig. 3 (a) Heat map and cluster dendogram showing differential gene expression between little spotted kiwi (LSK) and rowi. Green and red indicate low and high expression, respectively; data are normalized per gene and sample. Mean upregulation of genes in LSK relative to rowi (1753 genes) and vice versa (1331 genes) was $\sim 8$ and 5 fold and ranged from 2 to 701 fold per gene. (b) Biological process gene ontology (GO) assignments for genes significantly upregulated in LSK and rowi. Data are presented for GO terms representing 3\% or greater of assignments for at least one species and are weighted by the number of genes with $\mathrm{GO}$ terms per species

of 11.1 (range 2.0 to 101.4 , mean 12.2 where expression $>2$ fold). Of 150 genes differentially expressed between males and females, 83 (66 male-biased, 17 female-biased) could be mapped to a chicken chromosome; approximately $95 \%$ of these $(n=79)$ were syntenic to chrZ and none were syntenic to W (Fig. 4a; Additional file 8). The pattern of upregulation was particularly striking in males, with 64 genes syntenic to chicken chrZ and only two syntenic to other chromosomes. We were able to assign biological process GO terms to 52 genes showing malebiased expression and 21 genes exhibiting femalebiased expression. The distribution of genes across GO terms was similar between the sexes and to that observed between species.

Expression differences observed between species may reflect differences in the age of birds or their environment. All of our samples came from birds greater than one year of age and well past the timeframe of gonadal differentiation marking the onset of sex specific expression in other ratites (42 days) [35]. However, our LSK samples were largely from adult birds inhabiting three locations, our rowi samples were exclusively from juveniles ( 1 to 3 years old) inhabiting a fourth location, and gene expression can differ markedly with age and between environments, even after gonad differentiation $[36,37]$. Thus, the transcriptional variance observed between LSK and rowi could reflect physiological differences between adults and juveniles, environmental differences between the islands they occupy, or evolutionary divergence between the brown (including tokoeka (A. australis), North Island brown kiwi, and rowi) and spotted (including great spotted kiwi (A. haastii) and LSK) clades of kiwi [38]. In contrast, age and environmental effects cannot explain the observed sexbiased expression in LSK and rowi, because the sex ratio was equal within each age (three male and four female adults, five male and four female juveniles) and location (four male and four female rowi from Motuara Island, three male and three female LSK from Kapiti Island, one male and one female LSK from Long Island and one female LSK from Zealandia) sampled. Thus, differences in gene expression between the sexes likely reflect evolutionary differences between male and female kiwi.

Chickens and ratites exhibit strong chromosomal homology [35, 39-41] despite having diverged from a common ancestor 100 or more million years ago [30, 42, 43]. Indeed, highly conserved synteny among avian taxa allows chromosomal locations of kiwi genes to be predicted from that of chicken [35, 39]. In contrast, there is a huge diversity among birds in the degree of homomorphy and recombination between their $\mathrm{Z}$ and $\mathrm{W}$ chromosomes. The portions of the chromosomes that recombine define the PAR, where the same genes are present on both sex chromosomes and their sequences nearly identical [44]. Many avian lineages, including chicken, have highly differentiated sex chromosomes and a very small PAR, while more than two-thirds of the 
A

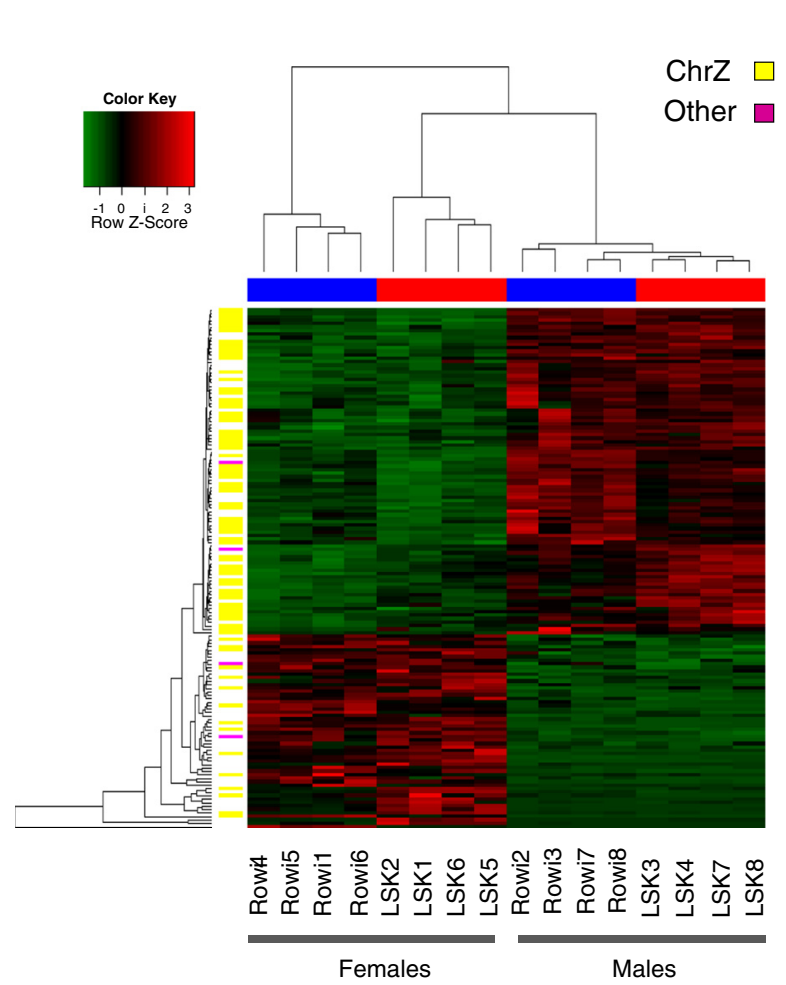

B
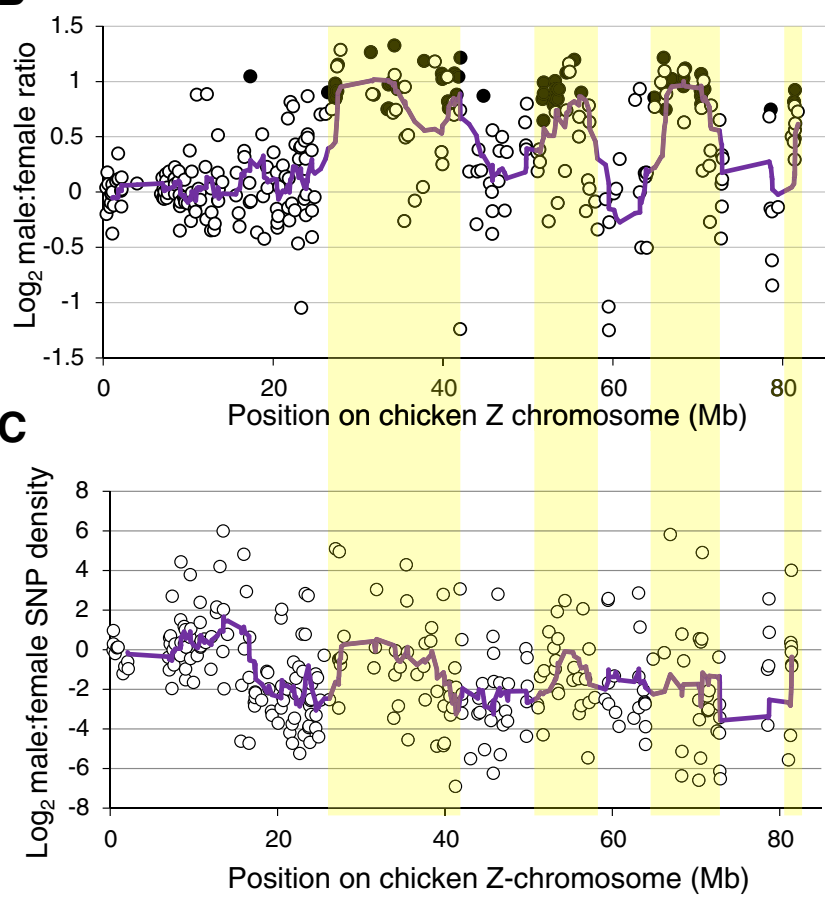

Fig. 4 a Heat map and cluster dendogram showing differential gene expression between male and female kiwi. Green and red indicate low and high expression, respectively; data are normalized per gene and sample. Mean male-biased (94 genes) and female-biased (56 genes) expression was 2 and 11 fold and ranged from 1 to 101. Sixty-four of 66 genes showing male-biased expression mapped to chicken chrZ (b) Distribution of male to female expression ratio (b) and SNP density (c) across the mapping position of chicken chrZ, including genes with significant malebiased expression (black circles) and genes equally expressed between sexes (open circles). Lines represent a moving average across ten genes; yellow shading indicates chromosomal regions with both male-biased expression and SNP density and so likely outside the PAR

emu (Dromaius novaehollandiae) and ostrich (Struthio camelus) $\mathrm{Z}$ chromosomes recombine with the $\mathrm{W}$ defining a very large PAR $[42,44,45]$. This pattern is not common to all ratites however, as the size of the PAR varies among tinamou species (Tinamus spp) and can represent as little as $1 \%$ of chrZ (white-throated tinamou, Tinamus guttatus) [44, 46]. The extent of homomorphy in kiwi sex chromosomes is unknown, and there is considerable scope for kiwi to differ from other ratites in sex chromosome arrangement since they diverged from their nearest extant ratite relatives, the emu and cassowary (Casuarius casuarius), approximately 58mya [47].

Given that dosage compensation is essentially lacking in birds and females are the heterogametic sex [42, 44, 48], genes on chrZ are expected to be expressed in males at equal (within the PAR) to twice (outside the PAR) the level observed in females. Similarly, SNP density is expected to be equal between the sexes at chrZ genes within the PAR, but male-biased in genes outside the PAR for which females are always hemizygous [35]. In line with these expectations, we found clear bimodal expression of genes mapped to chicken chrZ $(n=300)$, with a minority displaying two-fold male-biased expression $\left(\log _{2} \sim 1\right)$ and the majority displaying equal expression in males and females $\left(\log _{2} \sim 0\right.$; Fig. 4b). Regions of chrZ with male-biased expression also displayed male biased SNP density, which is consistent with these regions being outside of the PAR (Fig. 4c).

Upregulated kiwi genes formed disjunct clusters across the chicken chromosome. This clustering has been observed previously in ostriches and attributed to extensive PARs exhibiting strong recombination and homomorphy $[35,49]$ in conjunction with chromosomal inversions since the evolutionary split of paleognaths and neognaths [50]. Our data are consistent with these observations, and suggest that kiwi exhibit an ancestral state of sex chromosome differentiation similar to that of the ostrich and emu, with large PARs and little differentiation between the $\mathrm{Z}$ and $\mathrm{W}$ chromosomes $[35,44,49]$. The much greater mean expression among female-biased genes may be due to sexual antagonism [35]. Mutations on ratite sex chromosomes are more likely to fall within than outside the PAR. If these mutations are also sexually antagonistic, they cannot be restricted to the sex they benefit via restricted recombination between 
the sex chromosomes. Strongly sex-biased expression, however, can moderate the influence of these genes via upregulation in the sex they benefit, downregulation in the sex they harm, or both [35].

\section{Candidate genes}

Our data contain numerous functional genes that are potentially under selection in kiwi. For example, we have identified a kiwi gene that is homologous with chicken IGF1R (insulin-like growth factor 1 receptor) and associated with growth and body size in birds [51, 52], as well as seven forkhead box $(F O X)$ genes encoding transcription factors critical to proper development and healthy ageing [53-55]. The latter includes FOXC1 which is associated with ocular development and early onset glaucoma [56-58] and could be related to the unusually high frequency of cataracts in rowi (DKaye, pers comm).

Numerous kiwi genes likely associated with disease resistance were also discovered, including ovotransferrin (TF) [59], nine toll-like receptor genes (TLRs) [60, 61], and 15 tumor necrosis factor genes (TNFs) [62, 63]. TF encodes an iron-binding glycoprotein found in avian egg whites, serum, and eggshells that is associated with resistance to a wide variety of infections [64]. Similarly, $T L R$ genes function in pathogen recognition and innate immune responses [60]; they are targets of episodic and balancing selection in birds and highly polymorphic [61, 65]. TNF genes have roles in controlling inflammation, apoptosis, and cell proliferation and have been associated with numerous and varied diseases in vertebrates $[62,63]$. Variation within these genes could be a key component of fitness differences observed among kiwi. The first instances of avian malaria and small pox in kiwi were recently reported and kiwi management provides conditions that could promote the spread of infection via captive rearing of chicks and translocations of adults [66, 67]. TF and TLRs exhibit antimicrobial properties that could be critical for kiwi survival as well. Kiwi eggs have extremely high levels of antimicrobial proteins relative to other birds, including other ratites [68-70]. They also exhibit high levels of microbial contamination (over $90 \%$ of North Island brown kiwi eggs) with bacteria known to cause embryonic death in kiwi and other birds $[68,71]$. Thus, microbial infection of eggs and embryos could be a major cause of the hatching failure observed in wild kiwi $[21,72]$ and potentially have a genetic basis among individual kiwi as well.

Finally, our data also contain genes associated with important avian reproductive and behavioral traits. For example, GNRH1 (gonadotropin releasing hormone 1) is associated with double yolking [73], NPY2R (neuropeptide $Y$ receptor type 2) affects age at first lay and induces precocious puberty in chickens [73, 74], IGFII (insulinlike growth factor II) regulates avian ovarian function and egg productivity [75, 76], and OPN5 (opsin 5) encodes a UV sensitive photoreceptor protein that regulates seasonal reproduction in birds [77, 78]. Variation in these candidate genes may explain the extreme variation observed in reproductive success among the critically endangered rowi or among kiwi species generally. Similarly, exploring patterns of variation at several kiwi genes associated with important avian behaviors may shed light on kiwi ecology and conservation. These include DRD4 (dopamine receptor D4), which is associated with novelty seeking and exploratory behavior in birds and potentially related to colonization success [79], and FOXP1 (forkhead box P1), which is hypothesized to be involved in avian song learning and production [80].

\section{Conclusions}

Whole genome studies have been increasingly applied to species conservation since the advent of next generation sequencing $[5,6,81]$. For example, whole genome data have been used to determine that red (Canis rufus) and Great Lakes (C. lycaon) wolves arose from recent hybridization with other canids due to habitat changes and predator control efforts. This finding will likely shift the focus of conservation management from maintaining the admixed populations to conserving older, evolutionarily independent species [7]. Whole genome studies also recently found significant genetic population structuring and extensive inbreeding within species of great apes (Pan spp., Gorilla spp., and Pongo spp.) as well as pervasive hybridization among individuals in captivity [82]. These finding will provide the basis for genetic management of wild and captive great apes. Studies at the genomic level have also proven powerful for detecting selection and adaptation in endangered species. For example, rapid and positive selection has been shown for traits related to a predatory lifestyle in desert habitats in falcons (Falco spp.; beak shape, water conservation, sodium secretion) [8] and to diet shifts and olfaction in endangered giant pandas (Ursus maritimus) [9]. Finally, genomics has contributed to conservation via improving the resolution of historical demography studies. For example, Sumatran orangutans (Pongo abelii) currently have higher genetic diversity than Bornean orangutans (P. pygmaeus) despite a seven-fold lower population size. This pattern is apparently due to an exponential expansion of Sumatran orangutans and a decline in Bornean orangutans after their split $\sim 400$ thousand years ago [4].

Our study will allow a similar leap forward in conservation genomics of kiwi by providing a genome-wide resource to investigate their evolution and ecology and inform management. The transcriptome provides a critical complement to understanding an organism's genome. Thus, we expect the transcriptome assemblies provided here to be valuable in delineating the structure 
of rowi and LSK genomes once available. Our data contain autosomal and sex linked genetic markers representing both putatively neutral loci and loci under selection. This will vastly improve the current power of population genetics studies of kiwi which to date have been based on 30 microsatellite loci at most (KRamstad, unpublished data) $[24,34,83]$. Our new suite of markers will be used to test parentage, build pedigrees, and resolve relationships among birds relative to their mating and territorial behavior, as well as to improve estimates of inbreeding and the frequency of hybridization among kiwi. They will also improve the power of assignment tests for birds and eggs of unknown provenance, such as unlabeled museum specimens and those confiscated from illegal trade. The sex linked markers we have discovered (particularly those with sex-biased expression) may prove helpful to sex kiwi eggs, chicks, juveniles and remains which cannot be sexed phenotypically. Coupled with nuclear and mtDNA markers, they will also advance our understanding of sex specific dispersal patterns among kiwi, pseudoautosomal inheritance, and lack of dosage compensation in birds [35, 48, 49]. Finally, the numerous functional genes we have identified will allow powerful tests for genotype-phenotype associations in kiwi growth, development, disease resistance, reproduction, and behavior. Functional sequence variation in all of these candidate genes can now be assayed in hundreds of individual kiwi, bringing cutting-edge genomic techniques to bear on conservation of this ancient avian lineage.

\section{Methods}

\section{Sample collection, preparation, and sequencing}

Blood was collected from eight LSK and eight rowi (equal sex ratios). LSK were sampled from Kapiti Island (three males, two females), Long Island (one male, one female) and Zealandia Sanctuary (one female) populations. All LSK were adults when sampled apart from a single juvenile male from Kapiti Island. Adult LSK were sampled on Long Island in Austral autumn (2-4 April 2008) and outside of mating and nesting season in late winter and spring, [38] and all other LSK were sampled in Austral spring (23 Sept-15 Oct 2011) during mating and nesting season. Rowi sampled were part of the Operation Nest Egg program (ONE), which collects eggs from the only remaining wild population of rowi at Okarito Sanctuary, hatches them in captivity, rears them on predator-free Motuara Island until they reach sufficient body size to fend off predators, and then returns them as juveniles to Okarito Sanctuary. We sampled juvenile rowi ( 4 males, 4 females) inhabiting Motuara Island in Austral spring 2011 (4-6 October) and, thus, prior to breeding age and in the absence of predators or adult rowi. All but one bird was captured in the wild and in healthy condition when sampled. The exception was an emaciated female LSK that was sampled after being found trapped in a ground hole in Zealandia Sanctuary.

Approximately $0.5 \mathrm{ml}$ of whole blood was drawn from the metatarsal vein of each bird using a sterile $1 \mathrm{ml}$ syringe and 22-26 gauge needle, and added to $125 \mu \mathrm{l}$ $\mathrm{K}_{2}$ EDTA and $750 \mu$ of Trizol-LS. Total RNA was extracted from whole blood using a Trizol-LS extraction (Invitrogen) followed by a HighPure RNA Isolation clean up (Roche). After phase extraction with Trizol and chloroform, an equal volume of $75 \%$ ethanol was added to the aqueous phase and the whole mixture applied to a HighPure column; purification of RNA from the column was performed according to the manufacturer's instructions. Extracts were then treated with RNasin ribonuclease inhibitor (InVitro Technologies) to stabilize the RNA, followed by a DNase treatment to degrade any remaining DNA contamination.

Illumina sequencing was performed by New Zealand Genomics Ltd (Dunedin, New Zealand). RNA integrity was assessed with an Agilent Technologies 2100 Bioanalyzer, which indicated a mean RNA concentration of 64.6ng/ul (range 18-173) and a mean RNA Integrity Number (RIN) of 6.6 (range 6-7.5). Following purification via polyA selection, mRNA libraries were created using the TruSeq ${ }^{\mathrm{Tu}}$ RNA Sample Preparation Kit (Illumina, San Diego, CA, USA) and pooled in equimolar amounts before loading onto a total of four lanes of an Illumina HiSeq2000 sequencer for $1 \times 100 \mathrm{bp}$ paired end sequencing to a total depth of $851,254,015$ paired reads. Three rowi were initially sequenced to assess the level of hemoglobin transcripts followed by sequencing of the remaining 13 samples.

\section{De novo assembly and assembly annotation}

A small subset of the reads $(250,000)$ were first assembled using the Trinity package $[84,85]$. From the resulting contigs, two distinct sequences were identified that were expressed at a high level ( $20 \%$ of reads) and correlated to hemoglobin subunit genes. The entire dataset was then aligned to these contigs with Bowtie 2.0 [86] and the aligned reads removed. Remaining sequences were assembled de novo to create individual and composite transcriptome assemblies for LSK and rowi separately. All transcripts were filtered based on the primary transcript (noted seq1 in Trinity) to remove alternative splicing variants, and searched for an ORF using the inbuilt Trinity Open Read Frame finding script. Contigs with an ORF of greater than 100 amino acids were mapped against chicken genome protein sequences (Chicken Ensembl release78, http://uswest.ensembl.org/ Gallus_gallus) and orthologous genes were identified as the BLASTP hit with the highest score and alignment 
length. Chromosomal location of kiwi genes were predicted to be the same as that of their chicken orthologues based on highly conserved synteny across avian taxa $[35,39,41]$. To verify the integrity of our transcriptome assemblies, we aligned the composite de novo LSK assembly at the DNA level with the recently published North Island brown kiwi genome (ENA accession number: GCA 001039765.1) using BLAT [87].

The composite de novo LSK assembly was also BLAST searched against the NCBI NR protein database using BLASTx to return the top 20 non-redundant matches per sequence with an e-value cut-off of $1 \times 10^{-4}$. BLASTx results were then assigned GO labels in Blast2GO Pro ( $\mathrm{v}$ 2.7.2) [88] with an e-value cut-off of $1 \mathrm{e}^{-}{ }^{6}$, an annotation score cut-off of 55, and a GO weight of five. GO annotations were merged with those from an InterPro scan to further improve annotation, and grouped into GO-slim terms to simplify the output. Combined graphs of biological process and molecular function were then produced with nodes containing fewer than 10 sequences filtered out. BLASTx and Blast2GO outputs were uploaded into a MySQL database to facilitate calculation of summary statistics and searches for specific genes.

\section{SNP identification and validation}

All reads for each of the 16 individual birds were mapped as pairs to the LSK composite reference (20,152 genes containing ORF $>100$ amino acids) using Bowtie 2 [86]. Single nucleotide variants (SNPs) and small insertion/deletions were then identified using GATK version $1.2[89,90]$. To confidently identify SNPs that distinguish between species or individuals, we filtered the data for a minimum coverage of 50 reads across the variant in each of the birds and, within contigs where there was a density of less than 1 variant per 200bp, selected those markers where at least one individual had a SNP frequency lower than 5\% (expected reference genotype) and at least one individual had a SNP frequency greater than 35\% (expected heterozygous or homozygous genotype).

\section{Gene expression analysis}

We determined the raw expression of each transcript for each sample by mapping all reads back to the LSK reference assembly and counting the number of reads mapping to each transcript per sample in the Bowtie 2.0 alignment. We normalized the reads by quantile and assessed differential expression with $\beta$-statistics using functions in the EdgeR package [91]. Expression comparisons were made between species and between sexes. Differences between species were reported where differential expression was $\geq 2$ fold and $\beta$-statistics exceed zero. We did not use a 2 fold threshold in comparing expression differences between sexes, however, as we were specifically testing for 2 fold male-biased expression indicating genes present on the $\mathrm{Z}$ chromosome but outside the PAR. Results are given for differential expression between the sexes with and without the 2 fold threshold for comparison where appropriate.

\section{Additional files}

Additional file 1: FASTA file of LSK composite reference transcriptome. Includes 20,152 contigs containing an open reading frame greater than 100 amino acids. (FASTA $45736 \mathrm{~kb}$ )

Additional file 2: Comma separated file (.CSV) of BLAST results and alignment with the North Island brown kiwi genome. Includes the top BLAST hit for each LSK reference contig against NCBI-NR, chicken Ensembl, human Ensemb/ database, as well as the contig of the kiwi genome assembly to which each transcript mapped and the percent length mapped. (CSV $6520 \mathrm{~kb}$ )

Additional file 3: Comma separated file (.csv) of 66,909 SNPs that differentiate between LSK and rowi. Includes SNP position within the reference assembly, reference and alternative alleles, if SNP is untranslated or causes synonymous or non-synonymous substitutions, and predicted chicken chromosome synteny. (CSV 2607 kb)

Additional file 4: Comma separated file (.csv) of 12,384 SNPs that differentiate among LSK. Includes SNP position within the reference assembly, reference and alternative alleles, if SNP is untranslated or causes synonymous or non-synonymous substitutions, predicted chicken chromosome synteny, and the SNP frequency in each LSK sequenced. (CSV $921 \mathrm{~kb}$ )

Additional file 5: Comma separated file (.csv) of 29,313 SNPs that differentiate among rowi. Includes SNP position within the reference assembly, reference and alternative alleles, if SNP is untranslated or causes synonymous or non-synonymous substitutions, predicted chicken chromosome synteny, and the SNP frequency in each rowi sequenced. (CSV 2237 kb)

Additional file 6: Comma separated file (.csv) of raw expression levels. The number of reads mapped to each LSK reference contig for all kiwi sampled (CSV $1820 \mathrm{~kb})$

Additional file 7: Comma separated file (.csv) of 3084 transcripts differentially expressed between LSK and rowi. Includes the $\beta$-statistic, magnitude of expression difference, and direction of regulation (CSV $100 \mathrm{~kb}$ )

Additional file 8: Comma separated file (.csv) of 150 transcripts differentially expressed between male and female kiwi. Includes the $\beta$-statistic, magnitude of expression difference, direction of regulation (given in males relative to females), and synteny to the chicken genome (where available) (CSV $5 \mathrm{~kb}$ )

\section{Abbreviations}

chrZ: chromosome Z; DRD4: dopamine receptor D4; FOX: forkhead box; FOXC1: forkhead box C1; FOXP1: forkhead box P1; GNRH1: gonadotropin releasing hormone 1; GO: gene ontogeny; IGFIR: insulin-like growth factor 1 receptor; IGFII: insulin-like growth factor II; IUCN: International Union for Conservation of Nature; LSK: little spotted kiwi; mRNA: messenger RNA; mtDNA: mitochondrial DNA; NCBI NR database: National Center for Biotechnology Information non-redundant database; NPY2R: neuropeptide $Y$ receptor type 2; ONE: Operation Nest Egg; OPN5: opsin 5; ORF: open reading frame; PAR: pseudoautosomal region; SNP: single nucleotide polymorphism; TF: ovotransferrin; TLR: toll-like receptor; TNF: tumor necrosis factor.

\section{Acknowledgements}

We thank I Graham, D Kaye, R Colbourne, H Robertson and Zealandia Sanctuary for providing blood samples for this study. J Gardner and the Access Group kindly provided lab space and support, K Buckley, R Ramstad, T Lough, R Day, A Kumarasuriyar, and K Sluis provided sequencing and bioinformatics support, M Hasenbank designed and built the MySQL database, and New Zealand Genomics Ltd provided advice in study design and HiSeq sequencing. FW Allendorf, CH Daugherty, and HR Taylor provided helpful input on study design and draft manuscripts and the paper was 
improved by comments from one anonymous reviewer. This study was conducted in partnership and with guidance from Kaitiaki o Kapiti Trust, Te Rūnanaga Ngāi Tahu, Port Nicholson Block Settlement Trust, Te Ātiawa Manawhenua Ki Te Tau Ihu Trust, Te Rūnanga o Makaawhio, and Waiorua Bay Trust, and is dedicated to our colleague and collaborator I Jamieson.

\section{Funding}

Funding for this study was provided by Illumina Australia, New Zealand Genomics Ltd, New Zealand Ministry of Business, Innovation and Employment, and the Allan Wilson Centre.

\section{Availability of supporting data}

Raw HiSeq reads are available at the NCBI Short Read Archive under accession number SRP074490.

\section{Authors' contributions}

$\mathrm{KR}$ and HM designed the study. KR procured the samples, performed the RNA extractions and facilitated Illumina sequencing. GK performed the bioinformatic and statistical analyses, HM designed and facilitated production of the MySQL database, and KR conducted candidate gene and BLAST searching of transcriptomes. All authors contributed to drafting and editing the manuscript and have read and approve the final manuscript.

\section{Competing interests}

GK is an employee of Illumina, Inc, a public company that develops and markets systems for genetic analysis. KMR and HCM have no competing interests.

\section{Consent for publication}

We have permission for use of the single personal communication included.

\section{Ethics approval}

Handling and sampling of kiwi was carried out according to the Kiwi Best Practice Manual [38], under New Zealand Department of Conservation authority, with approval of the Victoria University of Wellington Animal Ethics Committee, and with guidance from the indigenous Māori communities of the sampling areas.

\section{Author details}

'Department of Biology and Geology, University of South Carolina Aiken, 471 University Parkway, Aiken, SC 29801, USA. ${ }^{2}$ Allan Wilson Centre, School of Biological Sciences, PO Box 600, Victoria University of Wellington, Wellington 6140, New Zealand. ${ }^{3}$ Biomatters Ltd, Level 2, 18 Shortland Street, Auckland Central, Auckland 1010, New Zealand. ${ }^{4}$ Illumina Australia, Scoresby, VIC, Australia.

Received: 27 October 2015 Accepted: 7 May 2016

Published online: 26 May 2016

\section{References}

1. Hoffman J, Thorne M, Trathan P, Forcada J. Transcriptome of the dead characterisation of immune genes and marker development from necropsy samples in a free-ranging marine mammal. BMC Genomics. 2013;14:52.

2. Hoffman Jl. Gene discovery in the Antarctic fur seal (Arctocephalus gazella) skin transcriptome. Mol Ecol Res. 2011:11:703-10.

3. Miller H, Biggs P, Voelckel C, Nelson N. De novo sequence assembly and characterisation of a partial transcriptome for an evolutionarily distinct reptile, the tuatara (Sphenodon punctatus). BMC Genomics. 2012;13:439.

4. Locke DP, Hillier LW, Warren WC, Worley KC, Nazareth LV, Muzny DM, et al. Comparative and demographic analysis of orang-utan genomes. Nature. 2011:469:529-33.

5. McMahon BJ, Teeling EC, Höglund J. How and why should we implement genomics into conservation? Evol Appl. 2014;7:999-1007.

6. Ryder O. Conservation genomics: applying whole genome studies to species conservation efforts. Cytogenet Genome Res. 2005;108:6-15.

7. von Holdt BM, Pollinger JP, Earl DA, Knowles JC, Boyko AR, Parker H, et al. A genome-wide perspective on the evolutionary history of enigmatic wolf-like canids. Genome Res. 2011:21:1294-305.

8. Zhan X, Pan S, Wang J, Dixon A, He J, Muller MG, et al. Peregrine and saker falcon genome sequences provide insights into evolution of a predatory lifestyle. Nat Genet. 2013;45:563-6.
9. Zhao S, Zheng P, Dong S, Zhan X, Wu Q, Guo X, et al. Whole-genome sequencing of giant pandas provides insights into demographic history and local adaptation. Nat Genet. 2013;45:67-71.

10. Vera JC, Wheat CW, Fescemyer HW, Frilander MJ, Crawford DL, Hanski I, et al. Rapid transcriptome characterization for a nonmodel organism using 454 pyrosequencing. Mol Ecol. 2008;17:1636-47.

11. Le Duc D, Renaud G, Krishnan A, Almén MS, Huynen L, Prohaska SJ, et al. Kiwi genome provides insights into evolution of a nocturnal lifestyle. Genome Biol. 2015;16:1-15.

12. Sales J. The endangered kiwi: a review. Folia Zool. 2005;54:1-20.

13. McCall R, Nee S, Harvey P. The role of wing length in the evolution of avian flightlessness. Evol Ecol. 1998;12:569-80.

14. Robertson HA, Colbourne RM. Survival of little spotted kiwi (Apteryx owenii) on Kapiti Island. Notornis. 2004:51:161-3.

15. Haussmann MF, Winkler DW, O'Reilly KM, Huntington CE, Nisbet IC, Vleck CM. Telomeres shorten more slowly in long-lived birds and mammals than in short-lived ones. Proc R Soc B. 2003:270:1387-92.

16. Holmes D, Flückiger R, Austad S. Comparative biology of aging in birds: an update. Exp Geront. 2001;36:869-83.

17. Martin GR. Sensory capacities and the nocturnal habit of owls (Strigiformes) Ibis. 1986:128:266-77.

18. IUCN. IUCN Red List of Threatened Species. 2015. www.iucnredlist.org Accessed 15 May 2016.

19. Burbidge $\mathrm{M}$, Colbourne R, Robertson H, Baker A. Molecular and other biological evidence supports the recognition of at least three species of brown kiwi. Cons Genet. 2003:4:167-77.

20. Holzapfel SA, Robertson HA, McLennan JA, Sporle W, Hackwell K, Impey M. Kiwi (Apteryx spp) recovery plan 2008-2018. Wellington: Department of Conservation; 2008. Report No 60.

21. Colbourne R, Bassett S, Billing T, McCormick H, McLennan J, Nelson A, et al. The development of Operation Nest Egg as a tool in the conservation management of kiwi. Wellington: Department of Conservation; 2005.

22. Miller HC, Bowker-Wright G, Kharkrang M, Ramstad KM. Characterisation of class II B MHC genes from a ratite bird, the little spotted kiwi (Apteryx owenii). Immunogenetics. 2011;63:223-33.

23. Taylor HR. Detecting inbreeding depression in a severely bottlenecked, recovering species: the little spotted kiwi (Apteryx owenii) [PhD]. Biological Sciences, Victoria University of Wellington. 2014

24. Ramstad KM, Colbourne RM, Robertson HA, Allendorf FW, Daugherty $\mathrm{CH}$. Genetic consequences of a century of protection: serial founder events and survival of the little spotted kiwi (Apteryx owenii). Proc $\mathrm{R}$ Soc B. 2013;280.

25. Briskie JV, Mackintosh M. Hatching failure increases with severity of population bottlenecks in birds. Proc Natl Acad Sci U S A. 2004;101:558-61.

26. Jamieson IG, Ryan CJ. Increased egg infertility associated with translocating inbred takahe (Porphyrio hochstetteri) to island refuges in New Zealand. Biol Conserv. 2000:94:107-14.

27. Keller LF, Waller DM. Inbreeding effects in wild populations. Trends Ecol Evol. 2002:17:230-41.

28. Sittmann K, Abplanalp H, Fraser RA. Inbreeding depression in Japanese quail. Genetics. 1966:54:371-9.

29. Westemeier RL, Brawn JD, Simpson SA, Esker TL, Jansen RW, Walk JW, et al. Tracking the long-term decline and recovery of an isolated population. Science. 1998;282:1695-8.

30. Subramanian S, Huynen L, Millar C, Lambert D. Next generation sequencing and analysis of a conserved transcriptome of New Zealand's kiwi. BMC Evol Biol. 2010;10:387.

31. Field LA, Jordan RM, Hadix JA, Dunn MA, Shriver CD, Ellsworth RE, et al. Functional identity of genes detectable in expression profiling assays following globin mRNA reduction of peripheral blood samples. Clin Biochem. 2007:40:499-502

32. Mastrokolias A, den Dunnen JT, van Ommen GB, 't Hoen PA, van RoonMom WM. Increased sensitivity of next generation sequencing-based expression profiling after globin reduction in human blood RNA. BMC Genomics. 2012;13:28

33. Shepherd L, Lambert D. Ancient DNA and conservation: lessons from the endangered kiwi of New Zealand. Mol Ecol. 2008;17:2174-84

34. Ramstad KM, Pfunder M, Robertson HA, Colbourne RM, Allendorf FW, Daugherty $\mathrm{CH}$. Fourteen microsatellite loci cross-amplify in all five kiwi species (Apteryx spp) and reveal extremely low genetic variation in little spotted kiwi (A. owenii). Cons Genet Res. 2010;2:333-6. 
35. Vicoso B, Kaiser VB, Bachtrog D. Sex-biased gene expression at homomorphic sex chromosomes in emus and its implication for sex chromosome evolution. Proc Natl Acad Sci. 2013;110:6453-8.

36. Fraser HB, Khaitovich P, Plotkin JB, Pääbo S, Eisen MB. Aging and gene expression in the primate brain. PLoS Biol. 2005;3:e274.

37. Whitney AR, Diehn M, Popper SJ, Alizadeh AA, Boldrick JC, Relman DA, et al. Individuality and variation in gene expression patterns in human blood. Proc Natl Acad Sci. 2003;100:1896-901.

38. Robertson H, Colbourne R, Castro I, Miller C, Cresswell M. Kiwi (Apteryx spp) best practice manual. Wellington: Department of Conservation; 2003.

39. Backström N, Karaiskou N, Leder EH, Gustafsson L, Primmer CR, Qvarnström A, et al. A gene-based genetic linkage map of the collared flycatcher (Ficedula albicollis) reveals extensive synteny and gene-order conservation during 100 million years of avian evolution. Genetics. 2008;179:1479-95.

40. Shetty S, Griffin D, Marshall GJ. Comparative painting reveals strong chromosome homology over 80 million years of bird evolution. Chromosome Res. 1999;7:289-95.

41. Zhang G, Li C, Li Q, Li B, Larkin DM, Lee C, et al. Comparative genomics reveals insights into avian genome evolution and adaptation. Science. 2014; 346:1311-20.

42. Ellegren $\mathrm{H}$. Evolution of the avian sex chromosomes and their role in sex determination. Trends Ecol Evol. 2000;15:188-92.

43. Jarvis ED, Mirarab S, Aberer AJ, Li B, Houde P, Li C, et al. Whole-genome analyses resolve early branches in the tree of life of modern birds. Science. 2014;346:1320-31.

44. Zhou Q, Zhang J, Bachtrog D, An N, Huang Q, Jarvis ED, et al. Complex evolutionary trajectories of sex chromosomes across bird taxa. Science. 2014;346:1246338.

45. Ogawa A, Murata K, Mizuno S. The location of Z-and W-linked marker genes and sequence on the homomorphic sex chromosomes of the ostrich and the emu. Proc Natl Acad Sci. 1998;95:4415-8.

46. Pigozzi Ml. Diverse stages of sex-chromosome differentiation in tinamid birds: evidence from crossover analysis in Eudromia elegans and Crypturellus tataupa. Genetica. 2011;139:771-7.

47. Mitchell KJ, Llamas B, Soubrier J, Rawlence NJ, Worthy TH, Wood J, et al. Ancient DNA reveals elephant birds and kiwi are sister taxa and clarifies ratite bird evolution. Science. 2014;344:898-900.

48. Itoh $Y$, Melamed E, Yang $X$, Kampf K, Wang $S$, Yehya N, et al. Dosage compensation is less effective in birds than in mammals. J Biol. 2007;6:2.

49. Adolfsson S, Ellegren $\mathrm{H}$. Lack of dosage compensation accompanies the arrested stage of sex chromosome evolution in ostriches. Mol Biol Evol. 2013; doi:10.1093/molbev/mst009.

50. Tsuda Y, Nishida-Umehara C, Ishijima J, Yamada K, Matsuda Y. Comparison of the Z and W sex chromosomal architectures in elegant crested tinamou (Eudromia elegans) and ostrich (Struthio camelus) and the process of sex chromosome differentiation in palaeognathous birds. Chromosoma. 2007;116:159-73.

51. Bennett A, Hester P, Spurlock D. Polymorphisms in vitamin D receptor, osteopontin, insulin-like growth factor 1 and insulin, and their associations with bone, egg and growth traits in a layer-broiler cross in chickens. Anim Genet. 2006;37:283-6.

52. Moe H, Shimogiri T, Kamihiraguma W, Isobe H, Kawabe K, Okamoto S, et al. Analysis of polymorphisms in the insulin-like growth factor 1 receptor (IGF1R) gene from Japanese quail selected for body weight. Anim Genet. 2007;38:659-61.

53. He Q, Morris BJ, Grove JS, Petrovitch H, Ross W, Masaki KH, et al. Shorter men live longer: association of height with longevity and $\mathrm{FOXO} 3$ genotype in American men of Japanese ancestry. PLoS ONE. 2014;9:e94385.

54. Morris BJ, Willcox D, Donlon T, Willcox B. FOXO3: a major gene for human longevity-a mini-review. Gerontology. 2015;61:515-25.

55. Palamaro L, Romano R, Fusco A, Giardino G, Gallo V, Pignata C. FOXN1 in organ development and human diseases. Int Rev Immunol. 2014;33:83-93.

56. Ito YA, Goping IS, Berry F, Walter MA. Dysfunction of the stress-responsive FOXC1 transcription factor contributes to the earlier-onset glaucoma observed in Axenfeld-Rieger syndrome patients. Cell Death Dis. 2014;5:e1069.

57. Lehmann OJ, Ebenezer ND, Jordan T, Fox M, Ocaka L, Payne A, et al. Chromosomal duplication involving the forkhead transcription factor gene FOXC1 causes iris hypoplasia and glaucoma. Am J Hum Genet. 2000;67:1129-35.

58. Tomonari S, Migita K, Takagi A, Noji S, Ohuchi H. Expression patterns of the opsin 5-related genes in the developing chicken retina. Dev Dyn. 2008;237: $1910-22$.

59. Giansanti F, Leboffe L, Pitari G, Ippoliti R, Antonini G. Physiological roles of ovotransferrin. Biochim Biophys Acta Gen Subj. 2012;1820:218-25.
60. Brownlie R, Allan B. Avian toll-like receptors. Cell Tissue Res. 2011;343:121-30.

61. Grueber CE, Wallis GP, Jamieson IG. Episodic positive selection in the evolution of avian toll-like receptor innate immunity genes. PLoS ONE. 2014; 9:e89632.

62. Aggarwal BB, Gupta SC, Kim JH. Historical perspectives on tumor necrosis factor and its superfamily: 25 years later, a golden journey. Blood. 2012;119:651-65.

63. Waters JP, Pober JS, Bradley JR. Tumour necrosis factor in infectious disease. J Pathol. 2013;230:132-47.

64. Giansanti F, Massucci MT, Giardi MF, Nozza F, Pulsinelli E, Nicolini C, et al. Antiviral activity of ovotransferrin derived peptides. Biochem Biophys Res Commun. 2005;331:69-73.

65. Grueber CE, Wallis GP, King TM, Jamieson IG. Variation at innate immunity toll-like receptor genes in a bottlenecked population of a New Zealand robin. PLoS ONE. 2012. doi:10.1371/journal.pone.0045011.

66. Ha H, Alley M, Howe L, Castro I, Gartrell B. Avipoxvirus infections in brown kiwi (Apteryx mantelli). N Z Vet J. 2013;61:49-52.

67. Howe L, Castro IC, Schoener ER, Hunter S, Barraclough RK, Alley MR. Malaria parasites (Plasmodium spp.) infecting introduced, native and endemic New Zealand birds. Parasitol Res. 2012;110:913-23.

68. Hiscox J. Can microbes be contributing to the decline of the North Island brown kiwi (Apteryx mantelli)? [MSc]. Zoology, Massey University. 2014

69. Osuga DT, Feeney RE. Biochemistry of the egg-white proteins of the ratite group. Arch Biochem Biophys. 1968;124:560-74.

70. Prager EM, Wilson AC, Arnheim N. Widespread distribution of lysozyme $g$ in egg white of birds. J Biol Chem. 1974;249:7295-7.

71. Wilson A. The triumphs, challenges and failures of young North Island brown kiwi (Apteryx matelli): A study of behaviour, growth, dispersal and mortality [PhD]. Zoology, Massey University. 2013.

72. McLennan J, Potter M, Robertson H, Wake G, Colbourne R, Dew L, et al. Role of predation in the decline of kiwi, Apteryx spp., in New Zealand. N Z J Ecol. 1996:20:27-35.

73. Dunn I, Miao Y, Morris A, Romanov MN, Wilson P, Waddington D. A study of association between genetic markers in candidate genes and reproductive traits in one generation of a commercial broiler breeder hen population. Heredity. 2004;92:128-34.

74. Fraley GS, Kuenzel WJ. Precocious puberty in chicks (Gallus domesticus) induced by central injections of neuropeptide Y. Life Sci. 1993;52:1649-56.

75. Kim M, Seo D, Ko Y. Relationship between egg productivity and insulin-like growth factor-l genotypes in Korean native Ogol chickens. Poult Sci. 2004;83: 1203-8.

76. Onagbesan O, Vleugels B, Buys N, Bruggeman V, Safi M, Decuypere E. Insulin-like growth factors in the regulation of avian ovarian functions. Domest Anim Endocrinol. 1999;17:299-313.

77. Kang SW, Kuenzel WJ. Deep-brain photoreceptors (DBPs) involved in the photoperiodic gonadal response in an avian species, Gallus gallus. Gen Comp Endocrinol. 2015;211:106-13.

78. Nakane Y, Ikegami K, Ono H, Yamamoto N, Yoshida S, Hirunagi K, et al. A mammalian neural tissue opsin (Opsin 5) is a deep brain photoreceptor in birds. Proc Natl Acad Sci. 2010;107:15264-8.

79. Mueller J, Edelaar P, Carrete M, Serrano D, Potti J, Blas J, et al. Behaviourrelated DRD4 polymorphisms in invasive bird populations. Mol Ecol. 2014;23: 2876-85.

80. Hara E, Perez JM, Whitney O, Chen Q, White SA, Wright TF. Neural FoxP2 and FoXP1 expression in the budgerigar, an avian species with adult vocal learning. Behav Brain Res. 2015;283:22-29.

81. Zhang G, Jarvis ED, Gilbert MTP. A flock of genomes. Science. 2014;346:1308-9.

82. Prado-Martinez J, Sudmant PH, Kidd JM, Li H, Kelley JL, Lorente-Galdos $B$, et al. Great ape genetic diversity and population history. Nature. 2013:499:471-5

83. Shepherd LD, Lambert DM. Nuclear microsatellite DNA markers for New Zealand kiwi (Apteryx spp). Mol Ecol. 2006;6:227-9.

84. Grabherr MG, Haas BJ, Yassour M, Levin JZ, Thompson DA, Amit I, et al. Fulllength transcriptome assembly from RNA-Seq data without a reference genome. Nat Biotechnol. 2011;29:644-52.

85. Haas BJ, Papanicolaou A, Yassour M, Grabherr M, Blood PD, Bowden J, et al. De novo transcript sequence reconstruction from RNA-seq using the Trinity platform for reference generation and analysis. Nat Protoc. 2013:8:1494-512.

86. Langmead B, Salzberg SL. Fast gapped-read alignment with Bowtie 2. Nat Meth. 2012:9:357-9.

87. Kent WJ. BLAT-the BLAST-like alignment tool. Genome Res. 2002;12:656-64. 
88. Conesa A, Götz S, García-Gómez JM, Terol J, Talón M, Robles M. Blast2GO: a universal tool for annotation, visualization and analysis in functional genomics research. Bioinformatics. 2005;21:3674-6.

89. McKenna A, Hanna M, Banks E, Sivachenko A, Cibulskis K, Kernytsky A, et al. The Genome Analysis Toolkit: a MapReduce framework for analyzing nextgeneration DNA sequencing data. Genome Res. 2010;20:1297-303.

90. Van der Auwera GA, Carneiro MO, Hartl C, Poplin R, del Angel G, Levy-Moonshine A et al. From FastQ data to high-confidence variant calls: the Genome Analysis Toolkit best practices pipeline. Curr Protoc Bioinformatics. 2013;doi:10.1002/ 0471250953.bi1110s43.

91. Robinson MD, McCarthy DJ, Smyth GK. edgeR: a Bioconductor package for differential expression analysis of digital gene expression data. Bioinformatics. 2010;26:139-40.

Submit your next manuscript to BioMed Central and we will help you at every step:

- We accept pre-submission inquiries

- Our selector tool helps you to find the most relevant journal

- We provide round the clock customer support

- Convenient online submission

- Thorough peer review

- Inclusion in PubMed and all major indexing services

- Maximum visibility for your research

Submit your manuscript at www.biomedcentral.com/submit
Biomed Central 